\title{
Physical and Genetic Mapping of the Salmonella dublin Virulence Plasmid pSDL2 Relationship to Plasmids from Other Salmonella Strains
}

Paul R. Beninger," Gary Chikami,* Ken Tanabe,* Chantal Roudier," Joshua Fierer,” and Donald G. Guiney*

*Department of Medicine, Division of Infectious Diseases H811F, University of California, San Diego Medical Center, San Diego, California 92103; and ${ }^{\ddagger}$ Departments of Medicine and Pathology, Veterans Administration Medical Center, San Diego, California 92161

\begin{abstract}
Plasmids of $\sim 80 \mathrm{~kb}$ in size are found in nearly all clinical isolates of Salmonella dublin and are believed to be essential for virulence. We have shown previously that the 80-kb plasmid pSDL2 is required for the $S$. dublin Lane strain to establish a lethal systemic infection in BALB/c mice after oral or intraperitoneal inoculation. We now present a physical and genetic characterization of pSDL2. We have established a complete restriction endonuclease cleavage map of pSDL2 for five enzymes: Xba I, Bam HI, Xho I, Sal I, and Hind III. The region specifying autonomous replication has been localized to a 10.5-kb region of the Sal I A fragment by subcloning on the vector pBR322. Using transposon insertion mutagenesis with Tn5-oriT, a region encoding the virulence phenotype has been mapped within a 6.4-kb portion of the Sal I B fragment. Deletions generated by partial Eco RI restriction digestion demonstrate that at least $50 \mathrm{~kb}$ of the plasmid DNA are not required for replication or virulence functions, confirming the map location of these phenotypes. Plasmids of different sizes and restriction patterns were found in mouse virulent strains of $S$. dublin $\mathrm{Vi}^{+}, S$. enteritidis, and $S$. choleraesuis. By Southern hybridization, these putative virulence plasmids share a common 4-kb Eco RI fragment with the virulence region of pSDL2, and the plasmids from $S$. dublin $\mathrm{Vi}^{+}$and $S$. enteritidis were shown to express mouse virulence comparable to pSDL2.
\end{abstract}

\section{Introduction}

Plasmid-encoded gene products are required for the virulence of many pathogenic Enterobacteriaceae that cause enteric or systemic disease (1). Plasmid-mediated virulence traits have been firmly established for Shigella (2-4) and Yersinia (5-7) species, as well as toxigenic and enteroinvasive strains of Escherichia coli $(8,9)$. More recently, plasmids in the 30-60-MD size range have been implicated in the virulence of nontyphoid Salmonella serotypes, including $S$. typhimurium (10-12), $S$. enteritidis $(13,14)$, and $S$. dublin $(11,15,16)$. In the case of $S$. dublin, a species that is host-adapted to cattle, nearly all clinical isolates contain plasmids of $\sim 80 \mathrm{~kb}(55 \mathrm{MD})(11,14-17)$, and a plasmid-free cured strain of $S$. dublin was shown by Terakado et al. (15) to have lost virulence for mice. Conclusive evidence for the role of plasmids in $S$. dublin virulence was provided by Chikami et al. (16), who labeled the plasmid from

Address all correspondence to Dr. Donald G. Guiney, Department of Medicine H81 1F, University of California, San Diego Medical Center, 225 Dickinson Street, San Diego, CA 92103.

Received for publication 9 March 1987 and in revised form 9 November 1987.

The Journal of Clinical Investigation, Inc.

Volume 81, May 1988, 1341-1347 a virulent $S$. dublin isolate with a derivative of the kanamycin transposon Tn5 and showed that reintroduction of the plasmid into both a cured strain and a naturally occurring plasmid-free isolate restored virulence for mice. Baird et al. (11) isolated two TnA transposon insertions that inactivated virulence in a similar $S$. dublin plasmid, and showed that this putative virulence region is homologous to an $S$. typhimurium plasmid. Current evidence suggests there is a family of virulence plasmids in Salmonella serotypes (17).

In both cattle and humans, $S$. dublin is particularly invasive, and oral infection frequently results in septicemia even without concomitant diarrhea (18-20). The course of infection that follows oral inoculation of $S$. dublin into BALB/c (ity $\left.{ }^{\mathrm{s}}\right)$ mice parallels the human infection, with colonization of the small bowel being followed by growth in Peyer's patches, mesenteric lymph nodes, and the spleen, ending in septicemia (16, 21). Heffernan et al. (21) compared the disease produced by the virulent, plasmid-containing $S$. dublin Lane strain with infection by a cured, plasmid-free derivative, designated LD842. Both Lane and LD842 colonized the bowel, multiplied in Peyer's patches, and spread to regional lymphatics and the spleen. However, only the Lane strain was able to cause progressive infection of the spleen and liver, while LD842 was contained by granuloma formation and eventually eliminated. Sublethal irradiation prevented the granulomatous inflammatory response and rendered the mice susceptible to infection with LD842, suggesting that mononuclear cells are essential for resistance to plasmid-free $S$. dublin. These in vivo results indicate that the virulence plasmid is not required for invasion of Peyer's patches nor for spreading to regional nodes and the spleen, but is necessary to establish a progressive infection of the reticuloendothelial system. Similar results have recently been reported for the virulence plasmids of $S$. typhimurium and a different isolate of $S$. dublin $(22,23)$.

This paper presents the physical and genetic characterization of the $S$. dublin virulence plasmid pSDL2. A restriction endonuclease cleavage map has been determined for five enzymes, and two important genetic functions have been mapped: the region specifying autonomous replication and the region encoding the virulence phenotype. Using a virulence region probe from pSDL2, the plasmids from $S$. dublin, $S$. enteritidis, and $S$. choleraesuis are shown to share a homologous 4-kb Eco RI fragment.

\section{Methods}

Growth media, bacterial strains, and plasmids. Trypticase soy broth (TSB) ${ }^{1}$ (BBL Microbiology Systems, Becton, Dickinson \& Co., Cockeysville MD), L-broth (LB), Mueller-Hinton medium, and M9 medium served as growth media (24). $S$. dublin Lane (a nicotinamide

1. Abbreviations used in this paper: LB, L-broth; ori T, origin of transfer; SSC, standard saline citrate; TSB, trypticase soy broth. 
auxotroph) is a clinical isolate from a blood culture, identified by standard microbiologic criteria (25), and contains the $80-\mathrm{kb}$ plasmid pSDL2 (16). LD842 is a derivative of $S$. dublin Lane from which pSDL2 has been cured (16). S. dublin 82/123, obtained from P. W. Jones, Compton, England, produces the Vi antigen and was identified and serogrouped by the California State Public Health Laboratory; the strain is auxotrophic for tryptophan. S. dublin 82/123 nal ${ }^{\mathrm{R}}$ was a spontaneous nalidixic acid-resistant mutant selected for growth on $100 \mu \mathrm{g} / \mathrm{ml}$ of nalidixic acid. S. enteritidis 697 is a clinical isolate from a patient with gastroenteritis. $S$. choleraesuis 116 was obtained from R. W. Griffith (26). E. coli JA221 (leuB, $\Delta t r p \mathrm{E} 5$, lacY, recA, hsd $\mathrm{R}^{-}$, $h s d \mathrm{M}^{+}$) and E. coli $\mathrm{C} 2110 \mathrm{nal}^{\mathrm{R}}$ ( pol A, his, rha) have been previously described $(27,28)$. For triparental matings, the helper plasmid was maintained in $E$. coli MV12, a C600 $\Delta$ trpE5, recA strain (29). The plasmid pBR322 Ap $\mathrm{Ap}^{\mathrm{r}} \mathrm{Tc}^{\mathrm{r}}$, a ColEl type-replicon, has been well characterized (30). pEYDG1 is a pBR322 replicon, with deletion of its own origin of transfer (ori $\mathrm{T}$ ) and $\mathrm{Tc}^{\mathrm{r}}$ gene, and carries a derivative of $\mathrm{Tn} 5$ which contains the oriT from the broad host range plasmid RK2 cloned into the Bam HI site of Tn5 without disruption of the kanamycin-resistance gene (31). The helper plasmid pRK2073 $\mathrm{Tp}^{\mathrm{R}} \mathrm{Sm}^{\mathrm{R}} \mathrm{tra}^{+}$is derived from pRK2013 by a Tn7 insertion in the kanamycin-resistance gene (31). pRK2013 was constructed by cloning the transfer region of RK2 together with kanamycin resistance into the Eco RI site of ColEl.

Restriction endonucleases. Xba I, Xho I, Bam HI, Sal I, Eco RI, and Hind III, and T4 DNA ligase were obtained from Bethesda Research Laboratories (BRL), Gaithersburg, MD. Reaction conditions conformed to the vendor's specifications.

Plasmid analysis. CsCl-purified plasmid DNA was prepared according to the method of Currier and Nester (32). Rapid isolation of plasmid DNA for clone analysis of Salmonella and $E$. coli strains was performed by a scaled-down modification of Currier and Nester's method as previously described (16).

Plasmid constructions and transformations. The replication region of pSDL2 was isolated by cloning in pBR322. Purified pSDL2 DNA $(\sim 0.5 \mu \mathrm{g})$ was completely digested with Sal I, mixed with $0.5 \mu \mathrm{g}$ of Sal I-digested pBR322 DNA, and ligated in a volume of $20 \mu$ l. The ligation mix (1-2 $\mu$ l) was transformed into $E$. coli $\mathrm{C} 2110$ using the standard $\mathrm{CaCl}_{2}$ technique originally described by Cohen et al. (33). The cells were plated on LB agar containing penicillin $(200 \mu \mathrm{g} / \mathrm{ml})$ and individual colonies were repicked onto penicillin plates, grown overnight in 5 $\mathrm{ml}$ of LB that contained penicillin, and analyzed for plasmid DNA (16). To isolate Eco RI deletions of pKT2 and pKT4, the plasmids were purified by $\mathrm{CsCl}$ /ethidium bromide density gradient centrifugation. Approximately $0.5 \mu \mathrm{g}$ of plasmid DNA was digested with Eco RI, then ligated in a volume of $100 \mu \mathrm{l}$ and transformed into $\mathrm{C} 2110$ selecting for penicillin resistance as described above. Eco RI deletions of the pSDL2::Tn5-ori T plasmids 9-18 and 16-11 were constructed similarly, except that partial Eco RI digestions were used for the ligations.

Isolation of Tn5-oriT insertions in Salmonella plasmids. The genetic procedures for isolating and characterizing Tn5-oriT transpositions into pSDL2 were modified from our previously published techniques (16). The manipulations are divided into five steps. (a) pEYDG1 (carrying the Tn5-oriT transposon) was transferred into $S$. dublin Lane containing pSDL2 by conjugation using the helper plasmid pRK2073. Strain E. coli JA221 (pEYDG1) was grown in LB with kanamycin $(50 \mu \mathrm{g} / \mathrm{ml})$, strain $E$. coli MV12 (pRK2073) was grown in Mueller-Hinton broth with trimethoprim $(100 \mu \mathrm{g} / \mathrm{ml})$, and $S$. dublin Lane was grown in LB. Strains were harvested in late log phase, washed once in normal saline, resuspended in normal saline to the original volume, and 1-ml vol of each were mixed together and passed through a $0.45-\mu \mathrm{m}$ filter (Millipore Continental Water Systems, Bedford, MA). The filter was incubated cell-side up on an LB agar plate at $37^{\circ} \mathrm{C}$ for 1 $h$. The bacteria were washed off the filter by vortexing in normal saline, and serial 10-fold dilutions were plated on M9-medium containing kanamycin $(50 \mu \mathrm{g} / \mathrm{ml})$ to select for $S$. dublin Lane containing pEYDG1. 100 colonies from these plates were repicked and tested for trimethoprim resistance ( $p R K 2073$ ); sensitive colonies were saved for the next step. (b) Transpositions of Tn5-oriT from pEYDG1 to pSDL2 were selected by a second conjugal transfer from $S$. dublin into $E$. coli C2110 (polA) nal ${ }^{\mathrm{R}}$ in which pSDL2::Tn5-oriT plasmids can replicate, but pEYDG1 and pRK2073 cannot replicate. A colony of $S$. dublin Lane containing both pSDL2 and pEYDG1 from step $a$ was grown to late $\log$ phase in LB containing kanamycin, washed, and mixed with $E$. coli MV12 (pRK2073) and E. coli $\mathrm{C} 2110$ nal $^{\mathrm{R}}$ according to the mating procedure described above. After mating, the cells were plated on LB agar that contained $50 \mu \mathrm{g} / \mathrm{ml}$ kanamycin and $25 \mu \mathrm{g} / \mathrm{ml}$ nalidixic acid to select for $\mathrm{C} 2110 \mathrm{nal}^{\mathrm{R}}$ colonies containing pSDL2::Tn5-oriT plasmids. (c) The pSDL2::Tn5-oriT plasmids were analyzed in C2110 by the rapid clone analysis procedure. Plasmid DNA was digested with Sal I and analyzed by agarose gel electrophoresis to locate the Tn5-oriT insertions within individual Sal I fragments. The precise location of the Tn5-ori T inserts in the Sal I B fragment was determined from Bam HI and Hind III digestions. (d) Individual pSDL2::Tn5-oriT plasmids designated for virulence testing were transformed into JA221, a trp ${ }^{-} E$. coli strain. (e) The pSDL2::Tn5-oriT plasmids in JA221 were transferred by conjugation into the plasmid-free, avirulent $S$. dublin LD842 strain, using the helper plasmid pRK2073. Midlog phase cells of JA221 (pSDL2::Tn5-oriT), MV12 (pRK2073), and S. dublin LD842 were harvested, washed, and mixed together as described above. After mating, LD842 colonies containing pSDL2::Tn5-oriT plasmids were selected on M9 agar with $100 \mu \mathrm{g} / \mathrm{ml}$ nicotinamide and $50 \mu \mathrm{g} / \mathrm{ml}$ kanamycin. These colonies were screened for trimethoprim resistance (pRK2073), and sensitive clones were saved for stability testing. A similar procedure was used to isolate and transfer Tn5-ori $\mathrm{T}$ insertions in pSDV1 and pSE1. Since $S$. dublin $82 / 123 \mathrm{Vi}^{+}$is a tryptophan auxotroph, the scheme was modified as follows: pEYDG1 was transferred into $S$. dublin $82 / 123 \mathrm{Vi}^{+}$nal $^{\mathrm{R}}$ using nalidixic acid $(20 \mu \mathrm{g} / \mathrm{ml})$ to counterselect against the $E$. coli donors. pSDV1::Tn5-oriT plasmids were then isolated by mating into $E$. coli $\mathrm{C} 2110$ with plating on M9 agar containing $50 \mu \mathrm{g} / \mathrm{ml}$ kanamycin.

In vitro stability testing. Each $S$. dublin LD842 strain containing a plasmid insertion mutant was tested for stability of the plasmid construct. The strain was grown in $\mathrm{LB}$ at $37^{\circ} \mathrm{C}$ without antibiotic selection for $\sim 30$ generations. The cells were diluted and replica plated on LB-agar and LB-agar + kanamycin. Stable mutants demonstrated $<2 \%$ loss. Unstable mutants demonstrated $>50 \%$ loss. About onehalf of the plasmids with Tn5-ori T inserts were unstable and could not be used for virulence testing.

Mouse infection. To test the virulence of $S$. dublin LD842 containing plasmid transposon insertions, strains were grown overnight with kanamycin selection $(50 \mu \mathrm{g} / \mathrm{ml})$ in TSB at $37^{\circ} \mathrm{C}$, washed in normal saline, and resuspended in TSB containing kanamycin to an optical density that corresponded to $10^{9} \mathrm{CFU} / \mathrm{ml}$. Each was further diluted in normal saline to $10^{4} \mathrm{CFU} / \mathrm{ml}$ just before inoculation, and $0.1 \mathrm{ml}$ was injected intraperitoneally, which represented $10^{2} \times \mathrm{LD}_{50}$ for $S$. dublin Lane (16). There were three or five female BALB/c (ity $)^{\mathrm{s}}$ ) mice (15-18 g) per group and groups were caged separately.

There was always a positive control group of mice injected intraperitoneally with $S$. dublin LD842 containing pSD6, a virulent Tn5ori $\mathrm{T}$ insertion derivative of pSDL2 (16). Mice were allowed access to food and water and observed for $14 \mathrm{~d}$. All positive control mice died between day 5 and day 8 . All survivors after $14 \mathrm{~d}$ were killed and their spleens were cultured in duplicate on TS agar and TS agar + kanamycin. Colony counts were within $10 \%$ for each pair of plates, and retention of the transposon-labeled plasmids was confirmed by the rapid plasmid analysis procedure.

DNA hybridization. The standard procedures for agarose gel electrophoresis and transfer of DNA fragments to nitrocellulose were performed as described previously (34). The virulence region probe consisted of the 4-kb Eco RI fragment contained within the Sal I B fragment of pSDL2 (see text and Fig. 4). The probe fragment was purified by agarose gel electrophoresis from an Eco RI digest of pSD15.2, which was constructed by ligating the Sal I B fragment of pSDL2 into the Sal I site of pBR322. The probe was labeled with biotin-7-dATP using nick-translation reagents from BRL. Hybridization, washes, and color 
development reactions were performed according to a protocol supplied by BRL (BluGene system). Hybridization was done overnight using $45 \%$ formamide ( $\mathrm{vol} / \mathrm{vol}$ ) at $42^{\circ} \mathrm{C}$, with successive washes in $2 \times$ standard saline citrate (SSC), $0.1 \%$ (wt/vol) SDS at room temperature; $0.2 \times$ SSC, $0.1 \%$ SDS at room temperature; and $0.16 \times$ SSC, $0.1 \%$ SDS at $50^{\circ} \mathrm{C}$.

\section{Results}

Endonuclease restriction map. We screened 18 different restriction enzymes for the number of sites present on pSDL2, and selected five enzymes (Xba I, Xho I, Bam HI, Sal I, and Hind III) for detailed mapping studies. Agarose gel electrophoretic patterns of pSDL2 digested with Xba I, Xho I, Bam HI, and Sal I are shown in Fig. 1. The restriction pattern of pSDL2 with Hind III has been published (16). There is a single Xba I site, three Xho I sites, three Bam HI sites, and seven Sal I sites (the third band of the Sal I digest is a doublet). Digestion with Hind III (not shown) results in nine sites (16). The Xba I site provides the reference point for constructing the restriction map shown in Fig. 2. A single digest of pSDL2 with Xho I was run in parallel with a double digest of PSDL2 with Xba I and Xho I. The 15.4-kb Xho I fragment is cleaved by Xba I into 12.0- and 3.4-kb subfragments. Similar parallel single and double digests oriented Xba I with respect to Bam HI, Sal I, and Hind III. A timed, partial digest of pSDL2 with Sal I identified the 6.5-kb $E$ and $F$ partial digest product, which showed that $E$ and $F$ are contiguous. The order of the Sal I B, D, E, and F fragments was determined from digestion of a large $36-\mathrm{kb} \mathrm{Bgl}$ II fragment which spans this region (not shown).

In the next step, the Sal I fragments were individually isolated on agarose gels. The Sal I A fragment, $28.5 \mathrm{~kb}$, contains a single Eco RI site, yielding subfragments of 18 and $10.5 \mathrm{~kb}$ (shown in Figs. 3 and 4). Restriction with Hind III resulted in

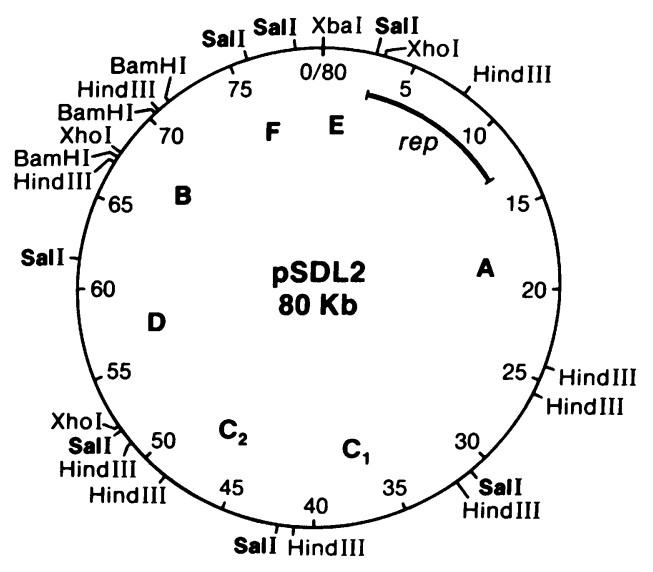

Figure 2. Endonuclease restriction map of pSDL2. The size is $80 \mathrm{~kb}$, which was derived from adding the sizes of individual fragments. It is oriented with respect to $\mathrm{Xba} \mathrm{I}$, a unique restriction site. Restriction sites are also shown for the three Xho I sites, three Bam HI sites, seven Sal I sites, and nine Hind III sites. rep represents the region associated with replication.

four subfragments: $16.2,5.7,5.2$, and $1.4 \mathrm{~kb}$. Comparison with the Hind III digest of pSDL2 and the large Eco RI-Sal I portion of the A fragment oriented the Hind III sites as shown in Figs. 2 and 4.

A similar strategy was employed with the Sal I B fragment. The distances from Xba I to the Bam HI, Hind III, and Xho I sites were determined by double digestions. The Sal I B fragment was gel purified and digested with Bam HI, Xho I, and Hind III. The internal Hind III G fragment was identified, as well as both internal Bam HI fragments. These two small Bam $\mathrm{HI}$ fragments (3.2 and $0.8 \mathrm{~kb}$ ) were purified and digested

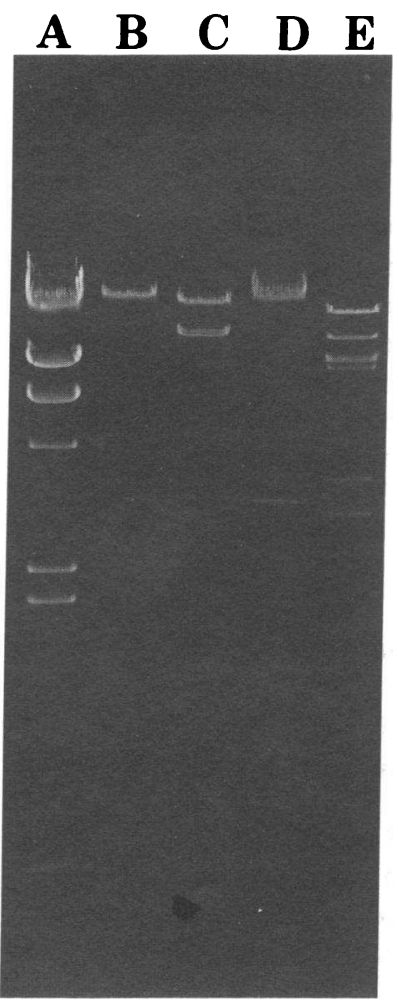

Figure 1. Agarose gel (0.7\%) electrophoretic patterns of the restriction endonuclease fragments of pSDL2. Lane $A$, a Hind III digest of lambda, serves as a size reference standard. Lanes $B, C, D$, and $E$ are digests of pSDL2 with Xba I, Xho I, Bam HI, and Sal I, respectively. The third largest Sal I band is a doublet.

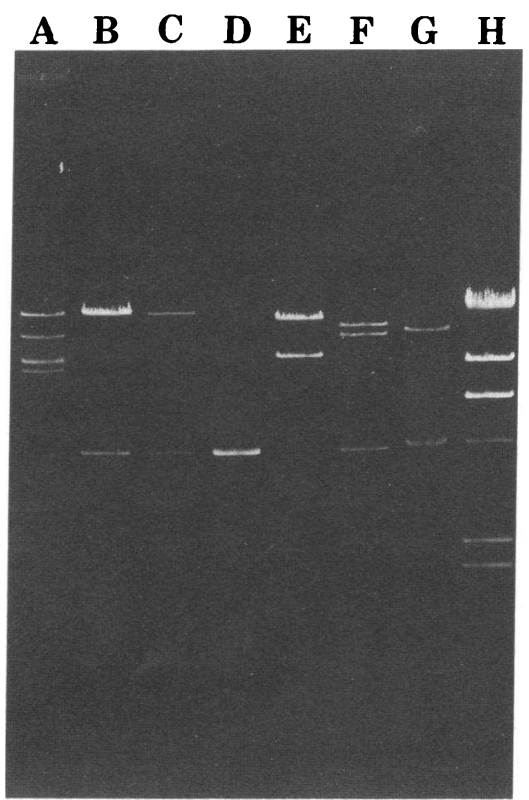

Figure 3. Agarose gel $(0.7 \%)$ electrophoretic patterns of restriction fragments from pKT2, pKT4, and pKT4 $\Delta 202$. Lane $A$, Sal I digest of pSDL2, showing the Sal I A, B, C ${ }_{1}+C_{2}, D$, $E$, and $F$ fragments in decreasing size; lane $B$, Sal I digest of pKT2; lane $C$, Sal I digest of pKT4; lane $D$, Sal I digest of pBR322; lane $E$, Eco RI digest of pKT2 showing the two fragments depicted in Fig. 4; lane $F$, Eco RI digest of pKT4 showing the presence of a unit length copy of pBR322 as well as the two Eco RI fragments derived from the Sal I A cloned fragment; lane $G$, Eco RI digest of pKT $4 \Delta 202$ showing loss of the large Eco RI fragment; and lane $H$, Hind III digest of lambda DNA. 


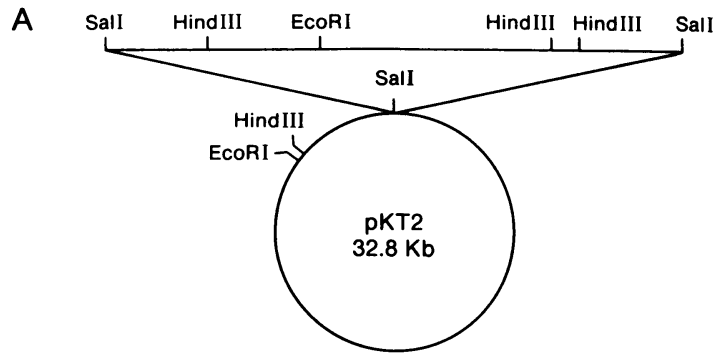

Scale (Kb)
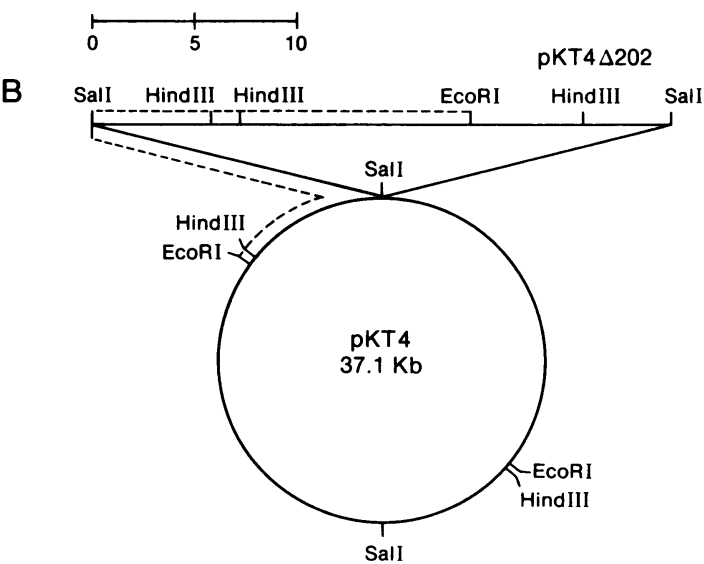

Figure 4. Endonuclease restriction maps of pKT2, pKT4, and pKT4 $\Delta 202$. These plasmids were selected for the ability to replicate in $E$. coli $\mathrm{C} 2110$, as described in the text. $(A)$ pKT2 is a $32.8-\mathrm{kb}$ plasmid containing the entire pSDL2 Sal I A fragment cloned into the unique Sal I site of pBR322. (B) pKT4 is a $37.1-\mathrm{kb}$ construct of pSDL2 Sal I A, with the opposite orientation to pKT2, cloned into a Sal I site of a dimer of pBR322. pKT4 4202 represents an Eco RI deletion clone, which further localized the region of replication to a Sal I-Eco RI 10.5-kb fragment. The dashed line indicates the DNA deleted.

with Xho I and Hind III to localize these sites. Using double digestions of purified fragments, the additional Xho I sites were shown to be just inside the Sal I A and Sal I D fragments. Since the order of the Sal I A, B, D, E, and F fragments is known, then Sal I $C_{1}$ and $C_{2}$ must lie next to each other between the $\mathrm{A}$ and $\mathrm{D}$ fragments. The Hind III sites in this region were determined by fragment purification and double digestions.

Isolation of the replication region of pSDL2. The region of pSDL2 that encodes autonomous replication and maintenance properties was isolated by subcloning on pBR322, a ColEl-type plasmid which is unable to replicate in the DNA polymerase I-deficient ( $p o l \mathrm{~A}$ ) strain $E$. coli $\mathrm{C} 2110$. Insertion of the region containing the replication system of pSDL2 into pBR322 allows the hybrid plasmid to replicate and express penicillin resistance in C2110. Accordingly, a complete Sal I digest of pSDL2 was ligated to Sal I-digested pBR322 and the mixture was transformed into $\mathrm{C} 2110$ selecting for penicillin resistance. Plasmids isolated from 24 separate resistant clones were digested with Sal I and examined by agarose gel electrophoresis. Most plasmids contained several Sal I fragments, but all plasmids had in common the Sal I A fragment in addition to pBR322. Two constructs containing only the Sal I A fragment and pBR322 were selected for further study and designated pKT2 and pKT4 (Figs. 3 and 4). Hind III and Eco RI restrictions of $\mathrm{pKT} 2$ and $\mathrm{pKT} 4$ demonstrated that the pSDL2
Sal I A fragment is in both of the two possible orientations with respect to pBR322, and that pKT4 contains an additional copy of pBR322. Using the unique Eco RI restriction site on pSDL2 Sal I A and the unique Eco RI site on pBR322, deletion clones were constructed. Using the same strategy described above, a complete Eco RI digest of either pKT2 or pKT4 was religated to itself, then the mixture was used to transform C2110, and the transformants were selected for penicillin resistance. None of the pKT2 penicillin-resistant transformants evaluated by clone analysis and Eco RI restriction had lost the smaller 10.5-kb Eco RI-Sal I portion of the Sal I A fragment from pKT2. However, transformants using pKT4 contained deletions of the larger 18-kb Eco RI-Sal I portion of the Sal I A fragment (Fig. 3). This deletion derivative, designated pKT4 2202 (Fig. 4), demonstrates that the remaining $10.5 \mathrm{~kb}$ of the Sal I A fragment contains the region of pSDL2 necessary for replication and maintenance of the plasmid.

Location of the virulence region of $p S D L 2$. We used transposon insertion mutagenesis to locate and map the virulence region on pSDL2. The Tn5-oriT transposon, a derivative of Tn5 containing the transfer origin of the conjugative plasmid RK2, is particularly useful because it allows mutated pSDL2 derivatives to be transferred by mating into $S$. dublin for virulence testing. Tn5-ori T insertions into pSDL2 were isolated as described in Methods. Selected mutants were transferred into the plasmid-free, avirulent $S$. dublin LD842 strain. 28 unique, stable Tn5-ori $\mathrm{T}$ mutants of pSDL2 were tested for virulence in mice by intraperitoneal inoculation of $10^{3}$ organisms. Two groups of mutant strains were clearly identified by these experiments: the virulent strains killed all the mice within $8 \mathrm{~d}$ while the avirulent strains killed none of the mice within $14 \mathrm{~d}$. Each avirulent strain was reisolated from the spleens of mice killed after $14 \mathrm{~d}$ and was shown to contain the pSDL2::Tn5ori T plasmid. This control showed that avirulence was due to the transposon insertion and not to loss of the plasmid in vivo. Mutants were initially selected for inserts that represented an even distribution about pSDL2, but once we had identified an avirulent mutant with its Tn5-ori T insert in the Sal I B fragment, we undertook an intensive effort to define the limits of that virulence region by selecting mutants with inserts concentrated in the Sal I B fragment. Fig. 5 represents a detailed map of the pSDL2 Sal I B fragment showing the Tn5-oriT inserts that were tested for virulence. Five mutants are avirulent. Their respective inserts are pSD15-1, 16-8, 13-4, 14-1, and $15-2$, which set an inner limit of $4.2 \mathrm{~kb}$ for the region encoding virulence. Two virulent inserts, pSD13-3 and 9-18, which are adjacent to the avirulent mutants pSD15-1 and 15-2 in the Sal I B fragment, set an outer limit of $6.4 \mathrm{~kb}$ for the virulence region. All of the other 23 mutants are virulent. The number of virulent inserts in the Sal I fragments are distributed as follows: Sal I A:5, Sal I B:5, Sal I C $1+2: 8$, Sal I D:3, Sal I $\mathrm{E}: 0$, and Sal I F:2.

Eco RI deletions of pSDL2 that replicate and express virulence. To confirm the location of the replication and virulence regions of pSDL2, we isolated a series of deletion derivatives generated by partial Eco RI digestion. We used two derivatives of pSDL2 that contained Tn5-oriT insertions adjacent to the virulence region: pSD9-18 and pSD16-11 (Fig. 5). These plasmids were partially digested with Eco RI, ligated, and transformed separately into $E$. coli JA221 selecting for kanamycin resistance. Several clones containing deletions were mapped and three are shown in Fig. 6. These derivatives were 

in the region required for virulence. $\bullet$, location of inserts that failed to abolish virulence (see text for Methods); O, inserts that abolished virulence expression. $E$, Eco RI; $H$, Hind III; $X$, Xho I; $B$, Bam HI; and $S$, Sal I. The Eco $R I$ sites were localized in this region by gel purification of the Sal I B fragment and selected subfragments followed by digestion with Eco RI.

transferred by conjugation into $S$. dublin LD842 and tested for virulence in mice as described in Methods. pSD9-18 $\Delta 11$ could not be maintained in LD842; all the kanamycin-resistant transconjugants that were analyzed contained Tn5-oriT in the chromosome. The two other constructs were stable in LD842 and restored the mouse virulence of this strain. Both pSD9-18 7 and pSD16-11 $\Delta 2$ lost $\sim 50 \mathrm{~kb}$ of DNA but retained stable replication and virulence properties as predicted from the cloning and Tn5-oriT insertion data in Figs. 4 and 5.

Homology between the virulence region of pSDL2 and plasmids in different strains of Salmonella. We had previously examined 16 different isolates of $S$. dublin and found plasmids similar to pSDL2 by size and restriction pattern in each strain (16). We subsequently analyzed an $S$. dublin isolate, designated $82 / 123$, that produced $\mathrm{Vi}$ antigen and had lower virulence in mice ( $\mathrm{LD}_{50}$ of $10^{3}$ by intraperitoneal injection) than $S$. dublin Lane. This strain contained a single plasmid of $60 \mathrm{~kb}$ (pSDV1) with a restriction pattern considerably different from pSDL2. In addition, we analyzed the mouse virulent strains $S$. enteritidis 696 and $S$. choleraesuis 116 for the presence of putative virulence plasmids. $S$. enteritidis 696 contained a single 60 -kb plasmid (pSE1) while $S$. choleraesuis had two plasmids of 36 and $8 \mathrm{~kb}$ (designated pSCH1 and pSCH2). Eco RI restriction digests of these plasmids together with pSDL2 are shown in Fig. $7 A$; pSDL2, pSDV1, pSE1, and pSCH1 differ substantially in restriction pattern, and $\mathrm{pSCH} 2$ contains a single Eco RI site. To examine the homology of these plasmids with the virulence region of $\mathrm{pSDL} 2$, the 4-kb Eco RI fragment defined by the avirulent Tn5-oriT inserts 15-1, 16-8, 13-4, and 14-1 (Fig. 5) was labeled and used as a hybridization probe with the Southern blot of the gel shown in Fig. $7 \mathrm{~A}$; the result, shown in Fig. $7 B$, demonstrates that pSDV1, pSE1, and pSCH 1 all contain 4-kb Eco RI fragments with strong homology to the virulence region of pSDL2. The small $S$. choleraesuis plasmid pSCH2 lacks homology.

A similar hybridization study was done using as a probe the 10.5-kb Eco RI-Sal I fragment from pKT4 $\Delta 202$, and encoding the replication region of pSDL2 (Fig. 4). Homologous sequences were found in each of the other Salmonella virulence plasmids tested: pSDV1, pSE1, and pSCH1, although in these plasmids the homologous Sal I fragment was $7.5 \mathrm{~kb}$ as opposed to $28 \mathrm{~kb}$ for the Sal I A fragment of pSDL2 (data not shown).

Virulence of pSDV1 and pSE1 in S. dublin LD842. Since pSDV1 and pSE1 contain sequences homologous to the virulence region of pSDL2, we studied the virulence conferred by these plasmids in LD842. We isolated Tn5-ori T inserts in both pSDV1 (pSDV213) and pSE1 (pSE707), then transferred the derivatives separately into LD 842 by conjugation. As shown in Table I, both pSDV213 and pSE707 render LD842 as virulent as the wild-type $S$. dublin Lane that contains pSDL2.

\section{Discussion}

The restriction endonuclease map of pSDL2 presented in Fig. 2 permits localization and genetic mapping of two important plasmid functions and provides the basis for comparison with other Salmonella plasmids. The map agrees well with the partial restriction map of sites in and around the Sal I B fragment of the $S$. dublin plasmid 2229, which was reported by Baird et al. (11), indicating close homology between these independent isolates from different continents. The Hind III restriction fragments of pSDL2 are similar or identical to the patterns of

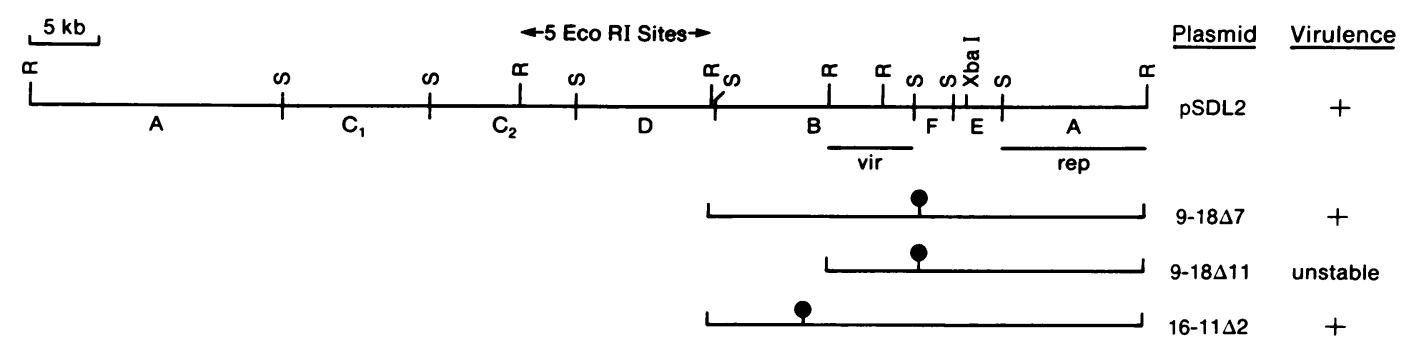

Figure 6. Map of Eco RIgenerated deletions of pSDL2. $R$, Eco RI sites; $S$, Sal I sites. The Sal I fragments are labeled $A$ to $F$ to correspond to the map in Fig. 2. The exact map location of a cluster of Eco RI sites in the $C_{2}$ and $D$ fragments has not been determined, but all of these sites have been lost in the deletion derivatives shown here. The sites of transposon insertions 9-18 and 16-11 (from Fig. 5) are shown as closed circles. Virulence was determined as described in Methods. 


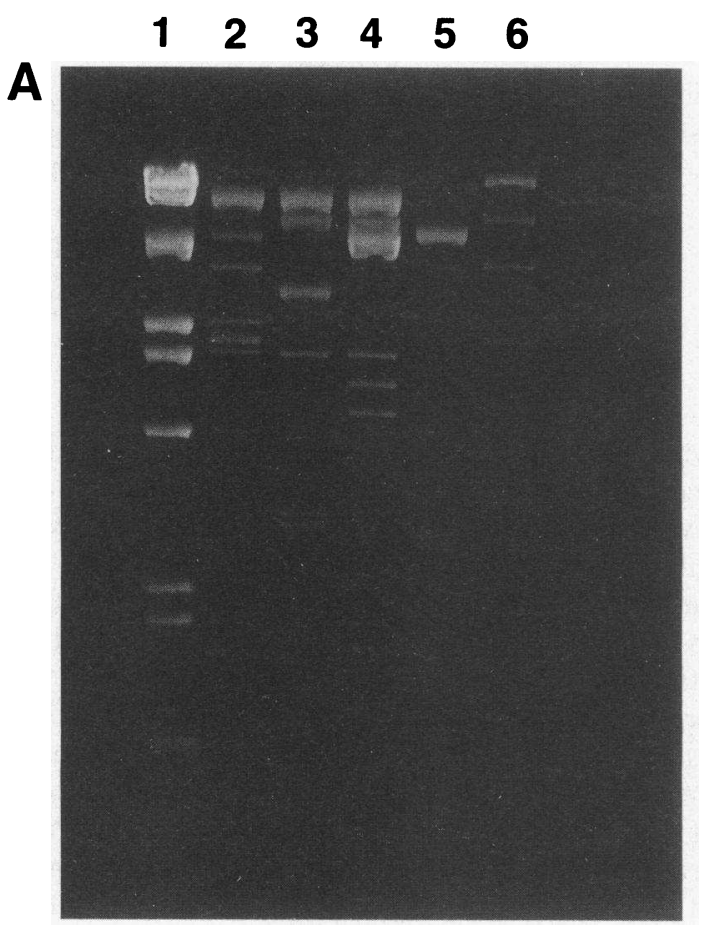

the Japanese isolates reported by Terakado et al. (15) and the French isolates of Popoff et al. (17), but differ from the $S$. dublin plasmids studied by Helmuth et al. (14). In addition to containing atypical plasmids, the $S$. dublin strains reported by Helmuth et al. have a different phenotype compared with strains isolated in California (25). Strains studied by Helmuth et al. are not auxotrophic, ferment arabinose, and are not lysed by phage $\mathrm{ES}_{18}$ (35; Fierer, J., unpublished results). Thus, there appears to be considerable conservation of sequences among certain $S$. dublin plasmids from different parts of the world, but more than one type of plasmid may exist.

The replication region of pSDL2 is located in the $10.5-\mathrm{kb}$ Sal I-Eco RI fragment shown in Figs. 2 and 4. Replication is a key function that determines the incompatibility, host range, and stable maintenance of the plasmid in different bacteria. pSDL2 is exceedingly stable in $S$. dublin (16). The replication fragment of pSDL2 hybridizes to regions in each of the other Salmonella virulence plasmids pSDV1, pSE1, and pSCH1,

Table I. Virulence of pSDVI and pSE1 in S. dublin LD842

\begin{tabular}{ccc}
\hline Strain & Dose & Deaths/total ${ }^{*}$ \\
\hline pSDV1 virulence & & \\
LD842 (pSDV213) & $2.4 \times 10$ & $3 / 3$ \\
& $2.4 \times 10^{2}$ & $3 / 3$ \\
S. dublin Lane (pSDL2) & $2.7 \times 10$ & $3 / 3$ \\
& $2.7 \times 10^{2}$ & $3 / 3$ \\
pSE1 virulence & & \\
LD842 (pSE707) & $3 \times 10$ & $4 / 5$ \\
& $3 \times 10^{2}$ & $5 / 5$ \\
S. dublin Lane (pSDL2) & $3 \times 10$ & $3 / 5$ \\
& $3 \times 10^{2}$ & $5 / 5$
\end{tabular}

* Mice were inoculated intraperitoneally and followed for $14 \mathrm{~d}$ as described in Methods.

\section{$\begin{array}{llllll}1 & 2 & 3 & 4 & 5 & 6\end{array}$}

B

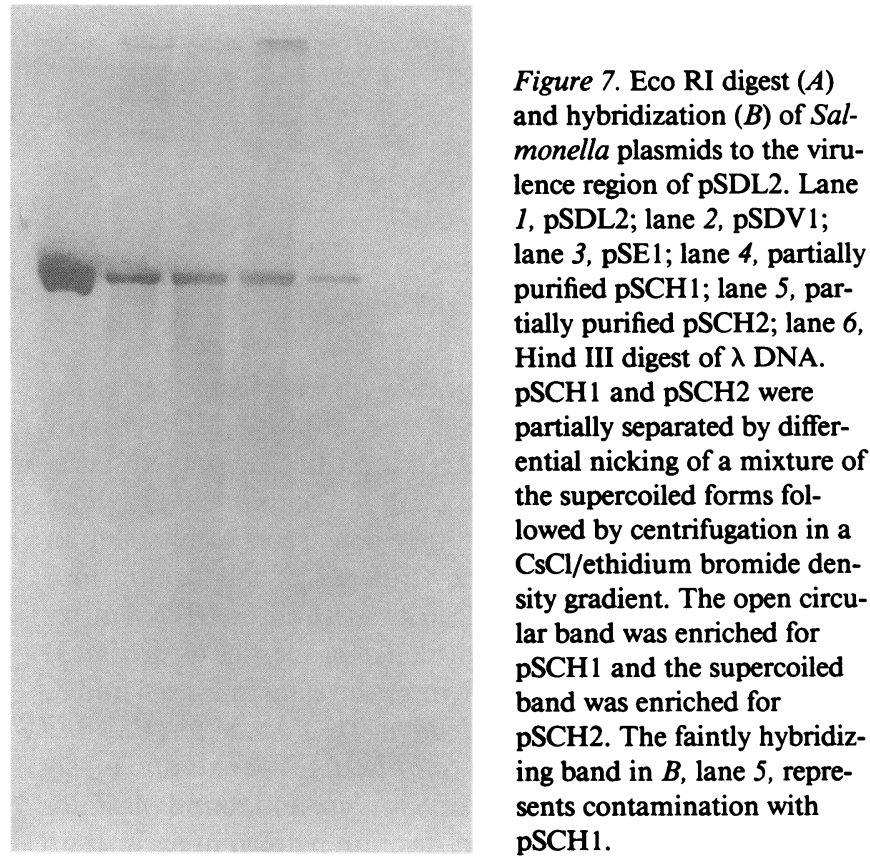

which suggests that these plasmids contain a common replication and maintenance system.

A region required for the virulence phenotype of pSDL2 is defined by the Tn5-oriT inserts shown in Fig. 5. The location of both the replication and virulence regions was confirmed by the isolation of the Eco RI deletions shown in Fig. 6. The virulence of plasmids $9-18 \Delta 7$ and $16-11 \Delta 2$ demonstrates that $\sim 50 \mathrm{~kb}$ of pSDL2 is not required for expression of the virulence phenotype. A similar result has recently been reported for the $90-\mathrm{kb}$ plasmid of $S$. typhimurium: virulence and primary replication functions map together in a $40-\mathrm{kb}$ region (36). Baird et al. (11) mapped two TnA transposon insertions that decrease virulence in their $S$. dublin strain 2229 plasmid. One insert is at about $72 \mathrm{~kb}$ on the map in Fig. 5, very close to the avirulent Tn5-oriT insert 14-1. The other avirulent TnA insertion was at about $66 \mathrm{~kb}$, within the Eco RI fragment that was deleted in pSD9-18 111 (Fig. 6). This TnA insertion could have affected the stability of their plasmid, since our results indicate that loss of this Eco RI fragment makes pSD9-18 11 very unstable in $S$. dublin.

The virulence region defined by the Tn5-ori $\mathrm{T}$ inserts is highly conserved among other Salmonella plasmids, as seen in Fig. 7. Despite differences in size and the pattern of restriction enzyme fragments, all of the plasmids studied contain a highly homologous 4-kb Eco RI fragment. This result suggested that these regions encoded similar virulence functions in the different plasmids. This hypothesis was confirmed when we were able to restore the virulence of LD842 by transfer of Tn5-oriT derivatives of $\mathrm{pSDV} 1$ and $\mathrm{pSE} 1$.

Strain $82 / 123$ is an unusual dublin isolate in that it produces $\mathrm{Vi}$ antigen, is a tryptophan auxotroph, and contains the pSDV1 plasmid with a considerably different restriction pattern compared with pSDL2 and 16 other $S$. dublin plasmids previously examined (16). However, the homology and mouse inoculation studies clearly show that pSDV1 encodes normal virulence properties in LD842. Furthermore, the ability to 
confer virulence on LD842 is not limited to plasmids of serotype dublin strains, since pSE1 from $S$. enteritiditis also renders LD842 virulent. These results suggest that despite differences in size and restriction enzyme digest patterns of plasmids isolated from different Salmonella serotypes, all these plasmids encode virulence functions by a common genetic mechanism.

\section{Acknowledgments}

This work was supported in part by Public Health Service grants GM-28924 and AI-16463 from the National Institutes of Health to Donald G. Guiney, a grant from the Veterans Administration Research Service to Joshua Fierer, and a Program Project PHS AM-35108 grant from the National Institutes of Health.

\section{References}

1. Elwell, L. P., and P. L. Shipley. 1980. Plasmid-mediated factors associated with virulence of bacteria to animals. Annu. Rev. Microbiol. 34:465-496.

2. Kopecko, D. J., O. Washington, and S. B. Formal. 1980. Genetic and physical evidence for plasmid control of Shigella sonnei form I cell surface antigen. Infect. Immun. 29:207-214.

3. Sansonetti, P. J., D. P. Kopecko, and S. B. Formal. 1982. Involvement of a plasmid in the invasive ability of Shigella flexneri. Infect. Immun. 35:852-860.

4. Watanabe, H., and K. N. Timmis. 1984. A small plasmid in Shigella dysenteriae 1 specifies one or more functions essential for $\mathrm{O}$ antigen production and bacterial virulence. Infect. Immun. 43:391396.

5. Gemski, P., J. R. Lazere, T. Casey, and J. A. Wohlhieter. 1980. Presence of a virulence-associated plasmid in Yersinia pseudotuberculosis. Infect. Immun. 28:1044-1047.

6. Ferber, D. M., and R. R. Brubaker. 1981. Plasmids in Yersinia pestis. Infect. Immun. 31:839-841.

7. Laroche, Y., M. van Bouchaute, and G. Cornelis. 1984. A restriction map of virulence plasmid pVYE439-80 from a serogroup 9 Yersinia enterocolitica strain. Plasmid. 12:67-70.

8. Smith, H. W., and S. Halls. 1968. The transmissible nature of the genetic factor in Escherichia coli that controls enterotoxin production. J. Gen. Microbiol. 52:319-334.

9. Harris, J. R., I. K. Wachsmuth, B. R. Davis, and M. L. Cohen. 1982. High-molecular weight plasmid correlated with Escherichia coli enteroinvasiveness. Infect. Immun. 37:1295-1298.

10. Jones, G. W., D. K. Rabert, D. M. Svinarich, and H. J. Whitfield. 1982. Association of adhesive, invasive, and virulent phenotypes of Salmonella typhimurium with autonomous 60-megadalton plasmids. Infect. Immun. 38:376-386.

11. Baird, G. D., E. J. Manning, and P. W. Jones. 1985. Evidence for related virulence sequences in plasmids of Salmonella dublin and Salmonella typhimurium. J. Gen. Microbiol. 131:1815-1823.

12. Fields, P. I., R. V. Swanson, C. G. Haidaris, and F. Heffron. 1986. Mutants of Salmonella typhimurium that cannot survive within the macrophage are avirulent. Proc. Natl. Acad. Sci. USA. 83:51895193.

13. Nakamura, M., S. Sato, T. Ohya, S. Suzuki, and S. Ikeda. 1985. Possible relationship of a 36-megadalton Salmonella enteritidis plasmid to virulence in mice. Infect. Immun. 47:831-833.

14. Helmuth, R., R. Stephan, C. Bunge, B. Hoog, A. Steinbeck, and E. Bulling. 1985. Epidemiology of virulence-associated plasmids and outer membrane protein patterns within seven common Salmonella serotypes. Infect. Immun. 48:175-182.

15. Terakado, N., T. Sehizaki, K. Hashimoto, and S. Naitoh. 1983. Correlation between the presence of a fifty-megadalton plasmid in Salmonella dublin and virulence for mice. Infect. Immun. 41:443-444.

16. Chikami, G. K., J. Fierer, and D. G. Guiney. 1985. Plasmidmediated virulence in Salmonella dublin demonstrated by use of a Tn5-ori T construct. Infect. Immun. 50:420-424.
17. Popoff, M. Y., I. Miras, C. Coynault, C. Lasselin, and P. Pardon. 1984. Molecular relationships between virulence plasmids of $\mathrm{Sal}$ monella serotypes typhimurium and dublin and large plasmids of other Salmonella serotypes. Ann. Microbiol. (Paris) 135A:389-398.

18. Hall, G. A., P. W. Jones, and M. M. Aitken. 1978. The pathogenesis of experimental intra-ruminal infections of cows with Salmonella dublin. J. Comp. Pathol. 88:409-417.

19. Werner, S. B., G. L. Humphrey, and I. Kamei. 1979. Association between raw milk and human Salmonella dublin infection. $\mathrm{Br}$. Med. J. 2:238-241.

20. Fierer, J. 1983. Invasive Salmonella dublin infections associated with drinking raw milk. West. J. Med. 138:665-669.

21. Heffernan, E. J., J. Fierer, G. Chikami, and D. Guiney. 1987. The natural history of oral Salmonella dublin infection in BALB/c mice: effect of a 80 kilobase-pair plasmid on virulence. J. Infect. Dis. 155:1254-1259.

22. Hackett, J., I. Kotlarski, V. Mathan, K. Francki, and D. Rowley. 1986. The colonization of Peyer's patches by a strain of Salmonella typhimurium cured of the cryptic plasmid. J. Infect. Dis. 153:11191125.

23. Manning, E. J., G. D. Baird, and P. W. Jones. 1986. The role of plasmid genes in the pathogenicity of Salmonella dublin. J. Med. Microbiol. 21:239-243.

24. Miller, J. H., editor. 1972. Experiments in Molecular Genetics. Cold Spring Harbor Laboratory, Cold Spring Harbor, New York. 431.

25. Fierer, J., and W. Fleming. 1983. Distinctive biochemical features of Salmonella dublin isolated in California. J. Clin. Microbiol. 17:552-554.

26. Griffith, R. W., T. T. Kramer, and J. F. Pohlenz. 1984. Relationship between antibody-complement susceptibility of smooth Salmonella choleraesuis var kunzendorf strains and their virulence for mice and pigs. Am. J. Vet. Res. 45:1342-1348.

27. Guiney, D. G., P. Hasegawa, and C. E. Davis. 1984. Plasmid transfer from Escherichia coli to Bacteroides fragilis: differential expression of antibiotic resistance phenotypes. Proc. Natl. Acad. Sci. USA. 81:7203-7206.

28. Sigetti, J. S., D. G. Guiney, and C. E. Davis. 1983. The mechanism of action of metronidazole in Bacteroides fragilis. J. Infect. Dis 148:1083-1089.

29. Hershfield, V., H. W. Boyer, C. Yanofsky, M. A. Lovett, and D. R. Helinski. 1974. Plasmid ColEl as a molecular vehicle for cloning and amplification of DNA. Proc. Natl. Acad. Sci. USA. 71:3455-3459.

30. Bolivar, R., R. L. Rodriguez, P. J. Greene, M. Belach, H. L. Heyneker, and H. W. Boyer. 1977. Construction and characterization of new cloning vehicles. II. A multipurpose cloning system. Gene (Amst.). 2:95-113.

31. Yakobson, E., and D. G. Guiney. 1984. Conjugal transfer of bacterial chromosomes mediated by the RK2 plasmid transfer origin cloned into transposon Tn5. J. Bacteriol. 160:451-453.

32. Currier, T. C., and E. W. Nester. 1976. Isolation of covalently closed circular DNA of high molecular weight from bacteria. Anal. Biochem. 76:431-441.

33. Cohen, S. M., A. Chang, and L. Hus. 1972. Nonchromosomal antibiotic resistance in bacteria: genetic transformation of Escherichia coli by R-factor DNA. Proc. Natl. Acad. Sci. USA. 69:2110-2114.

34. Chikami, G., D. G. Guiney, T. Schmidhauser, and D. R. Helinski. 1985. Comparison of 10 IncP plasmids: homology in the regions involved in plasmid replication. J. Bacteriol. 162:656-660.

35. LeMinor, L., and A. M. Chalen. 1975. Susceptibilité au bacteriophage $\mathrm{ES}_{18}$ de cultures de Salmonella dublin, S. enteritidis, S. blegdam et de sérotypes apparentés. Ann. Microbiol. (Paris). 126A:327331.

36. Michiels, T., M. Y. Popoff, S. Durviaux, C. Coynault, and G. Cornelius. 1987. A new method for the physical and genetic mapping of large plasmids: application to the localisation of the virulence determinants on the $90 \mathrm{~kb}$ plasmid of Salmonella typhimurium. Microb. Pathog. 3:109-116. 